\title{
EFFECTIVE THERMAL CONDUCTIVITY OF SPHERICAL PARTICULATE NANOCOMPOSITES: COMPARISON WITH THEORETICAL MODELS, MONTE CARLO SIMULATIONS AND EXPERIMENTS
}

\author{
HATIM MACHRAFI*, GEORGY LEBON \\ Thermodynamics of Irreversible Phenomena, University of Liège, Allée du 6-Août, 17 \\ Liège, 4000, Belgium \\ *H.Machrafi@ulg.ac.be \\ Received Day Month Year \\ Revised Day Month Year
}

\begin{abstract}
The purpose of this work is to study heat conduction in systems that are composed out of spherical micro-and nanoparticles dispersed in a bulk matrix. Special emphasis will be put on the dependence of the effective heat conductivity on various selected parameters as dimension and density of particles, interface interaction with the matrix. This is achieved by combining the effective medium approximation and extended irreversible thermodynamics, whose main feature is to elevate the heat flux vector to the status of independent variable. The model is illustrated by three examples: Silicium-Germanium, Silicaepoxy-resin and Copper-Silicium systems. Predictions of our model are in good agreement with other theoretical models, Monte-Carlo simulations and experimental data.
\end{abstract}

Keywords: Thermal conductivity, Composite materials, Extended Thermodynamics, Nanoparticles, Phonon dispersion.

\section{Introduction}

We investigate in this work some particular properties of heat conduction in nanocomposites. Nanocomposites know a huge variety of applications, such as heat conduction enhancement in polyesters (see Ref. 1) or energy storage systems (see Ref. 2), to mention a few. The change in thermal conductivity has also been exploited to increase the figure of merit $\mathrm{ZT}$ of thermoelectric materials which behaves as the inverse of the heat conductivity. ${ }^{3}$ The nanocomposite is generally made out of a homogeneous matrix in which nanoparticles are dispersed. For particles with diameters of the same order of magnitude or smaller than the phonon mean free path, the Fourier theory, based on the classical approach of thermodynamics, is not able to predict the heat flux thermal interactions between the matrix and the nanoparticles. Therefore, we propose to investigate the problem of heat conduction in nanocomposites by a more sophisticated thermodynamic formalism, namely Extended Irreversible Thermodynamics (EIT) ${ }^{4}$ In this approach, the heat flux is elevated to the status of independent variable at the same footing as the temperature. EIT has been successfully applied to transient heat transport in nanofilms in a previous paper. ${ }^{5}$

The main problem investigated in the present work is the determination of the effective heat conductivity for several nanocomposites. We will make use of the effective-medium approach (see Ref. 6) which provides a process of homogenization of the heterogeneous medium formed by the matrix and the particles. The basic formula for the effective heat conductivity coefficient $k^{e f f}$ is Maxwell's relation (see Refs. 7 and 8)

$k^{e f f}=k_{m} \frac{2 k_{m}+(1+2 \alpha) k_{p}+2 \varphi\left[(1-\alpha) k_{p}-k_{m}\right]}{2 k_{m}+(1+2 \alpha) k_{p}-\varphi\left[(1-\alpha) k_{p}-k_{m}\right]}$. 
In this expression, $k_{m}$ and $k_{p}$ designate the heat conductivities of the matrix and the suspended particles respectively, $\varphi$ is the volume fraction of the particles and $\alpha$ is a dimensionless parameter describing the particle-matrix interaction:

$\alpha=R k_{m} / a_{p, s}$.

The quantity $R$ is the thermal boundary resistance coefficient (see Ref. 9) given by

$R=4 / C_{m} v_{m}+4 / C_{p} v_{p}$

while $a_{p, s}$ is the so-called "specular" radius defined by

$a_{p, s}=a_{p}(1+s) /(1-s)$,

with $a_{p}$ the radius of the nanoparticle. The symbols $C_{m}$, $v_{m}, C_{p}$ and $v_{p}$ in (3) stand for the specific heat capacities and group velocities of the bulk matrix and the particles, respectively. In expression (4) of $a_{p, s}$, the symbol $s(0 \leq s \leq 1)$ denotes the surface specularity of the nano-particles, expressing the probability of specular scattering of phonons on the particle-matrix interface. For $s=0$, the surface is called diffuse, meaning that the direction of phonons after impact is independent of the direction of the impacting phonons, in which case $a_{p, s}$ is simply the particle radius $a_{p}$. For $s \rightarrow 1$, we have a surface on which the impacting phonons influence the direction of the out coming phonons and the surface is said to be perfectly specular. Note that the result (3) was established in the case of diffusive surfaces. ${ }^{10}$

An expression for $k_{m}$ is established in the framework of the effective-medium approach (see Refs. 6, 9, 11 and 12), while $k_{p}$ will be derived from EIT. In the next section, we propose expressions for the matrix and particle thermal conductivities and discuss the use of EIT. Our model will be applied to a SiliciumGermanium system (Si-Ge), comparing it to MonteCarlo simulations from the literature. Subsequently, the model is compared to two theoretical models for a Copper-Silicium $(\mathrm{Cu}-\mathrm{Si})$ mixture and finally the results for a Silica-epoxy-resin system $\left(\mathrm{SiO}_{2}\right.$-epoxy-resin) are faced to experimental results.

\section{Methods: Thermal Conductivity by Extended Irreversible Thermodynamics}

For the heat conductivity of the matrix, we use the classical Boltzmann-Peierls phonon expression

$k_{m}=\left.\frac{1}{3}\left(C_{m} v_{m} \Lambda_{m}\right)\right|_{T_{r e f}}$.

$T_{r e f}$ is the reference temperature, say the room temperature. Within the matrix, the phonons experience phonon-phonon interactions and the mean free path is given by the Matthiessen rule:

$\frac{1}{\Lambda_{m}}=\frac{1}{\Lambda_{m, b}}+\frac{1}{\Lambda_{m, \text { coll }}}$

with $\Lambda_{m, b}$ designating the mean free path in the bulk and $\Lambda_{m \text {,coll }}$ the supplementary contribution due to the interactions at the particle-matrix interface given by (see Ref. 9)

$\Lambda_{m, \text { coll }}=4 a_{p, s} / 3 \varphi$.

Concerning the derivation of $k_{p}$, we propose a new closed-form formula

$k_{p}=k_{p}^{0} f(K n, s)$,

wherein $k_{p}^{0}$ is the value of the thermal conductivity for the bulk material of which the particle is composed of and given by an expression similar to (5):

$k_{p}^{0}=\left.\frac{1}{3}\left(C_{p} v_{p} \Lambda_{p, b}\right)\right|_{T_{r e f}}$,

the quantity $f(K n, s)$ a correction factor, taking into account the dimension of the nanoparticles and their specularity, $K n$ is the Knudsen number defined as the ratio of the mean free path of the phonons inside the particle $\Lambda_{p, b}$, and the "specular" radius $\mathrm{a}_{p, s}$ of the particle:

$K n=\Lambda_{p, b} / \mathrm{a}_{p, s}$.

Expression (9) is analogous to that used for the matrix with the exception that now the mean free path is the bulk one (so that $\Lambda_{p}=\Lambda_{p, b}$ ), the contribution of the collisions being hidden in the correction factor $f(K n)$. The latter will be determined by referring to EIT. 
Effective Thermal Conductivity of Spherical Particulates Nanocomposites: Comparison with Theoretical Models, Monte Carlo Simulations and Experiments

At nanoscales, heat transport is mostly influenced by non-local effects. Non-local effects are introduced in the framework of EIT by appealing to a hierarchy of fluxes $\boldsymbol{Q}^{(1)}, \boldsymbol{Q}^{(2)}, \ldots, \boldsymbol{Q}^{(n)}$ with $\boldsymbol{Q}^{(1)}$ being identified with the heat flux vector $\boldsymbol{q}, \boldsymbol{Q}^{(2)}$ (a tensor of rank two) being the flux of the heat flux, $\boldsymbol{Q}^{(3)}$ the flux of $\boldsymbol{Q}^{(2)}$ and so on. From the kinetic theory point of view, the quantities $\boldsymbol{Q}^{(2)}$ and $\boldsymbol{Q}^{(3)}$ represent the higher moments of the velocity distribution. Omitting non-linear contributions in the fluxes, the hierarchy of equations can be cast in the form (see for more details chapter 4 in Ref. 4)

$$
\begin{aligned}
& \nabla T^{-1}-\alpha_{1} \partial_{t} \boldsymbol{Q}^{(1)}+\beta_{1} \nabla \cdot \boldsymbol{Q}^{(2)}=\mu_{1} \boldsymbol{Q}^{(1)}, \\
& \beta_{n-1} \nabla \boldsymbol{Q}^{(n-1)}-\alpha_{n} \partial_{t} \boldsymbol{Q}^{(n)}+\beta_{n} \nabla \cdot \boldsymbol{Q}^{(n+1)}=\mu_{n} \boldsymbol{Q}^{(n)}, \\
& (n=2,3, \ldots),
\end{aligned}
$$

where $\partial_{t}$ stands for the time derivative, and $\alpha_{i}, \beta_{i}$ and $\mu_{i}$ are phenomenological coefficients related to the relaxation times, correlation lengths and transport coefficients, respectively. Equation (11) reduces to the well-known law of Cattaneo (see Ref. 13) when the term $\nabla \cdot Q^{(2)}$ is omitted. We now consider an infinite number of flux variables $(n \rightarrow \infty)$ and apply the spatial Fourier transform

$$
\widehat{\boldsymbol{q}}(\boldsymbol{k}, t)=\int_{-\infty}^{+\infty} \boldsymbol{q}(\boldsymbol{r}, t) e^{-i \boldsymbol{k} \cdot \boldsymbol{r}} d \boldsymbol{r}
$$

to Eqs. (11) and (12), with $\widehat{\boldsymbol{q}}$ the Fourier transform of $\boldsymbol{q}$, $\boldsymbol{r}$ the spatial variable, $t$ the time and $\boldsymbol{k}$ the wavenumber vector. This procedure results into obtaining the following time-evolution equation for the heat flux:

$\bar{\tau}(\boldsymbol{k}) \partial_{t} \widehat{\boldsymbol{q}}(\boldsymbol{k}, t)+\widehat{\boldsymbol{q}}(\boldsymbol{k}, t)=-i \boldsymbol{k} k_{p}(\boldsymbol{k}) \widehat{T}(\boldsymbol{k}, t)$

where $\bar{\tau}(\boldsymbol{k})=\alpha_{1} / \mu_{1}$ designates a renormalized relaxation time depending generally on $\boldsymbol{k} . k_{p}(\boldsymbol{k})$ is given by the continued-fraction for the $\boldsymbol{k}$-dependent effective thermal conductivity:

$$
k_{p}(\boldsymbol{k})=\frac{k_{p}^{0}}{1+\frac{\boldsymbol{k}^{2} l_{1}^{2}}{1+\frac{\boldsymbol{k}^{2} l_{2}^{2}}{1+\boldsymbol{k}^{2} l_{3}^{2}}}},
$$

with $k_{p}^{0}$ the classical bulk thermal conductivity, given by Eq. (9), independent of the dimension of the system, $l_{n}$ is the correlation length of order $n$ defined by $l_{n}^{2}=$ $\beta_{n}^{2} /\left(\mu_{n} \mu_{n+1}\right)$. Here, it is assumed that the relaxation times $\tau_{n}(n>1)$ corresponding to higher order fluxes are negligible with respect to $\tau_{1}$, which is a hypothesis generally admitted in kinetic theories. In the present problem, there is only one dimension, namely the radius $a_{p, s}$ of the spheres, so that it is natural to define $k \equiv$ $2 \pi / a_{p, s}$. The correlation lengths selected as $l_{n}^{2}=$ $a_{n+1} l^{2}$, with $a_{n}=n^{2} /\left(4 n^{2}-1\right)$ and $l$ identified as the mean free path independently of the order of approximation. This is a rather natural choice for phonons as shown by Dreyer and Struchtrup. ${ }^{14}$ With these resukts in mind, the continued fraction (15) reduces to an asymptotic limit (see Ref. 15), leading finally to the following expression for $k_{p}$ :

$k_{p}=\frac{3 k_{p}^{0}}{4 \pi^{2} K n^{2}}\left[\frac{2 \pi K n}{\arctan (2 \pi K n)}-1\right]$.

\section{Results}

In this section, the model is applied to the $\mathrm{Si}-\mathrm{Ge}$ and $\mathrm{Cu}$-Si composites and $\mathrm{SiO}_{2}$-epoxy-resin. The results are compared to Monte Carlo simulations, theoretical models and experimental results.

\subsection{Si-Ge Composite; Comparison with Monte Carlo Simulations}

The model is firstly applied to $\mathrm{Si}$ spherical nanoparticles dispersed into a Ge matrix. The values for the specific heat capacity, phonon group velocity and bulk mean free path for these two components are given in Table 1, evaluated at room temperature. Most of the values in this table are obtained from the so-called dispersion model as proposed by Chen. ${ }^{10}$

Table1: Phonon properties for bulk materials at room temperature $\left(T_{r e f}\right)$

\begin{tabular}{lllll}
\hline Material & $\begin{array}{l}\text { Heat } \\
\text { capacity } \\
{\left[\mathrm{MJ} /\left(\mathrm{m}^{3} \mathrm{~K}\right)\right]}\end{array}$ & $\begin{array}{l}\text { Group } \\
\text { velocity } \\
{[\mathrm{m} / \mathrm{s}]}\end{array}$ & $\begin{array}{l}\text { Mean } \\
\text { path } \\
{[\mathrm{nm}]}\end{array}$ & free \\
\hline $\mathrm{Si}$ & $0.93^{\mathrm{a}}$ & $1804^{\mathrm{a}}$ & $268^{\mathrm{a}}$ & \\
$\mathrm{Ge}$ & $0.87^{\mathrm{a}}$ & $1042^{\mathrm{a}}$ & $171^{\mathrm{a}}$ & \\
$\mathrm{SiO}_{2}$ & $1.687^{\mathrm{b}}$ & $4400^{\mathrm{b}}$ & $0.558^{\mathrm{b}}$ & \\
$\mathrm{Epoxy}$ resin & $1.91^{\mathrm{c}}$ & $2400^{\mathrm{c}}$ & $0.11^{\mathrm{d}}$ & \\
$\mathrm{Cu}$ & $3.47^{\mathrm{e}}$ & $7723^{\mathrm{f}}$ & $45^{\mathrm{g}}$ & \\
\hline
\end{tabular}

${ }^{\text {aRefs. } 11,12 \text { and } 16}$ 
${ }^{\mathrm{b}}$ Ref. 17

${ }^{\mathrm{c}}$ Ref. 18

${ }^{\mathrm{d}}$ Ref. 10

${ }^{\text {eRef. } 19}$

${ }^{\mathrm{f} C a l c u l a t e d}$ from $v=3 k / C \Lambda_{b}$ by considering $k=402 \mathrm{Wm}^{-}$ ${ }^{1} \mathrm{~K}^{-1}$ from Ref. 20

${ }^{\text {gRef. }} 20$

For this example, we consider four values of the particle radius $a_{p}(5,25,50$ and $100 \mathrm{~nm})$ and four values for the specularity parameter $s(0,0.2,0.5$ and 1$)$, note that all the values of the particles radii are smaller than the mean free path of phonons in $\mathrm{Si}$. The results for the effective thermal conductivity as a function of the volume fraction of the nanoparticle are reported on Figs. 1 through 4. Results from Monte-Carlo simulations (see Ref. 21) are shown for comparison for particle radii $a_{p}$ of 5, 25 and $100 \mathrm{~nm}$. Although the $\mathrm{Si}$ nanoparticles have a larger bulk heat conductivity than the matrix Ge, one observes a decrease of the effective thermal conductivity of the whole system, whereas one should expect an increase. However, according to (16), the main cause of variation of the thermal conductivity is, besides the thermal boundary resistance of the interface, the dimension of the nanoparticles: the smaller is the radius, the smaller is the effective heat conductivity.

Comparison with values from the literature (see Refs. 9 and 11) shows a good correspondence for several particle radii and surface specularities, except for large particle radii and high values of the specularity parameter for which higher effective thermal conductivities are predicted. Monte-Carlo simulations indicate a good agreement with our model for specularities ranging from $s=0$ to $s=0.2$, depending on the particle radius. This implies that for $\mathrm{Si}-\mathrm{Ge}$ systems, the specularity parameter should be approximately between $s=0$ and $s=0.2$, so that a rather diffuse surface is expected.

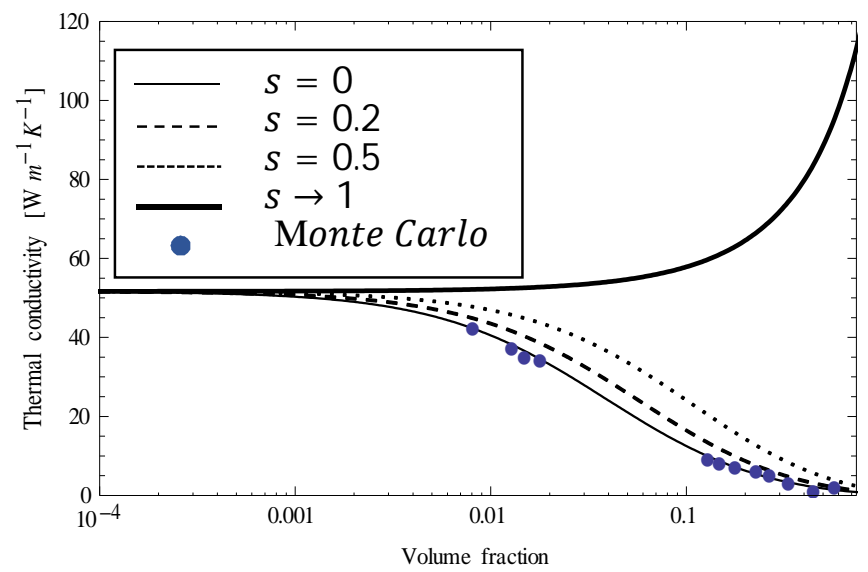

Fig. 1: Effective thermal conductivity of the nanocomposite $\mathrm{Si}-\mathrm{Ge}$ as a function of the volume fraction $(\varphi)$ of $\mathrm{Si}$ particles for different $s$ values with radius: $a_{p}=5 \mathrm{~nm}$. Monte Carlo simulations (see Ref. 21) are shown in filled circles.

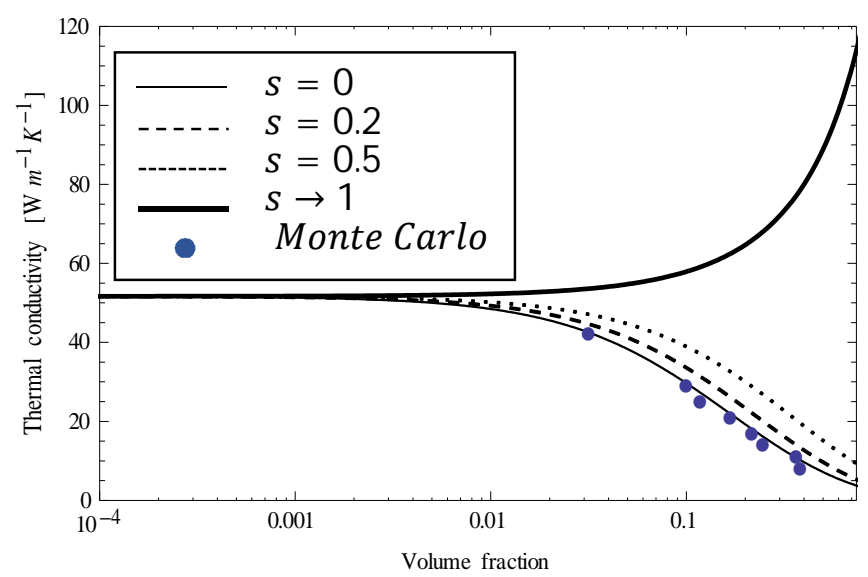

Fig. 2: Effective thermal conductivity of the nanocomposite $\mathrm{Si}-\mathrm{Ge}$ as a function of the volume fraction $(\varphi)$ of Si particles for different $s$ values with radius: $a_{p}=25 \mathrm{~nm}$. Monte Carlo simulations (see Ref. 21) are shown in filled circles. 
Effective Thermal Conductivity of Spherical Particulates Nanocomposites: Comparison with Theoretical Models, Monte Carlo Simulations and Experiments

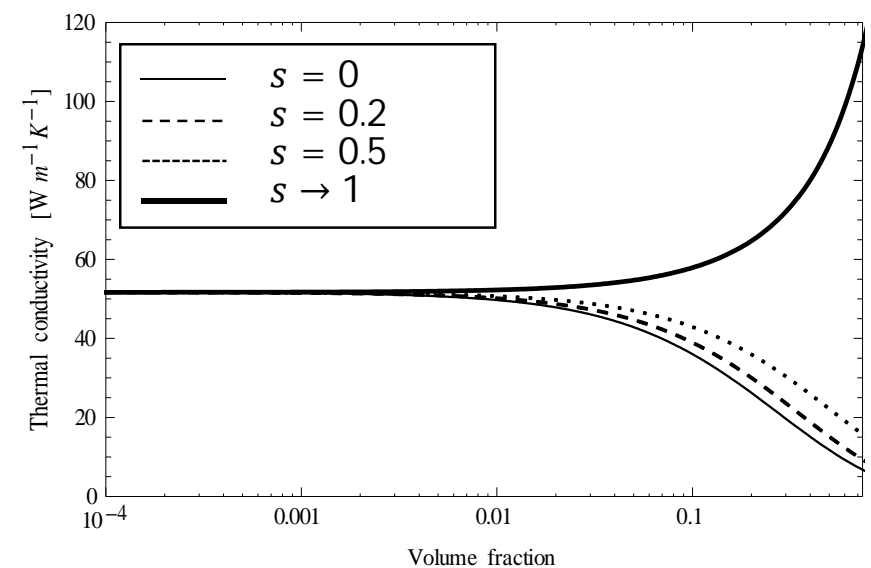

Fig. 3: Effective thermal conductivity of the nanocomposite $\mathrm{Si}-\mathrm{Ge}$ as a function of the volume fraction $(\varphi)$ of $\mathrm{Si}$ particles for different $s$ values with radius: $a_{p}=50 \mathrm{~nm}$.

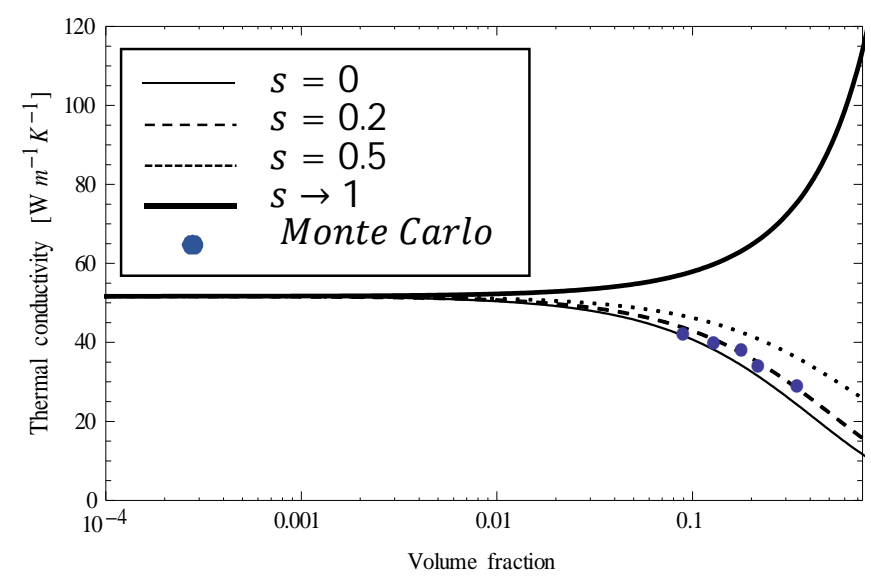

Fig. 4: Effective thermal conductivity of the nanocomposite $\mathrm{Si}-\mathrm{Ge}$ as a function of the volume fraction $(\varphi)$ of Si particles for different $s$ values with radius: $a_{p}=100 \mathrm{~nm}$. Monte Carlo simulations (see Ref. 21) are shown in filled circles.

\subsection{Cu-Si Composite; Comparison with Recent Models}

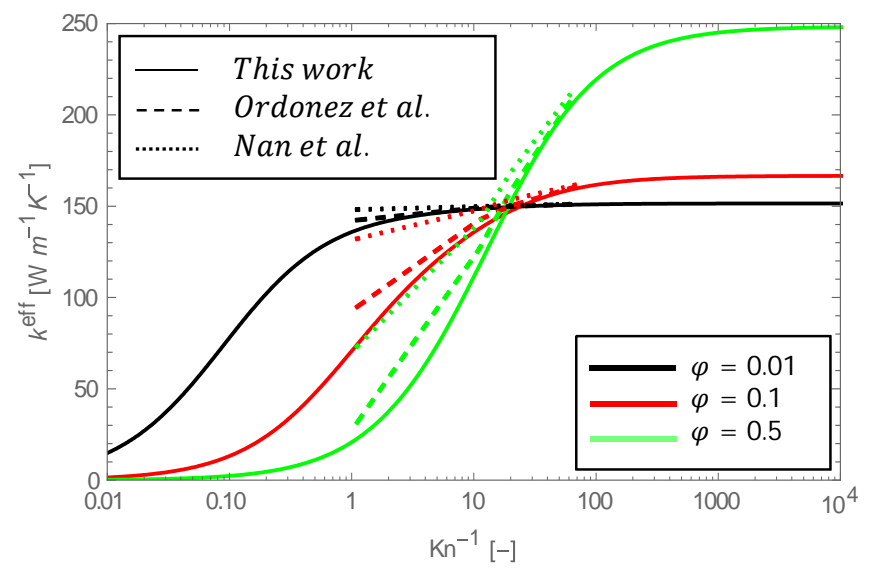

Fig. 5: Effective thermal conductivity of the nanocomposite $\mathrm{Cu}-\mathrm{Si}$ as a function of the volume fraction $(\varphi)$ of $\mathrm{Cu}$ particles for different particle radii: $a_{p}=50$, 500, 900 and $3000 \mathrm{~nm}(s=0)$. Comparison with theoretical models (see Refs. 6 and 20).

A further check of the validity of the model is provided by calculating the effective heat conductivity of a different material, namely $\mathrm{Cu}$ particles dispersed in a $\mathrm{Si}$ matrix, see Fig. 5 where the results of our approach are compared with those of Nan et al. (see Ref. 6) and Ordonez and Alvarado (see Ref. 20). The values for the specific heat capacity, phonon group velocity and bulk mean free path for $\mathrm{Cu}$ and $\mathrm{Si}$ are given in Table 1, four values of the particle radius are considered $\left(a_{p}=50\right.$, 500,900 and $3000 \mathrm{~nm}$ ), ranging from nano- to microdimensions. The specularity parameter is $s=0$. It is observed that the agreement with Ordonez and Alvarado (see Ref. 20) is satisfactory but weak concerning Nan et al. (see Ref. 6), especially for small particles $\left(a_{p}<500 \mathrm{~nm}\right)$. The expression for $k^{\text {eff }}$ obtained by Nan et al. is based on Fourier's law whose validity is questionable at small length scales. It is worth to stress that our model leads to good results, even within the Fourier limit.

\subsection{SiO2-Epoxy Resin; Comparison with Experimental Results}

The system with $\mathrm{SiO}_{2}$ nanoparticles embedded in an epoxy resin is investigated because it offers the opportunity to compare with experimental data. ${ }^{22}$ The values for the specific heat capacity, phonon group velocity and bulk mean free path for $\mathrm{SiO}_{2}$ and the epoxy resin are given in Table 1. Fig. 6 shows the results for 
$a_{p}=10 \mathrm{~nm}$, while Fig. 7 presents a zoom for volume fractions between $\varphi=0$ and $\varphi=0.1$.

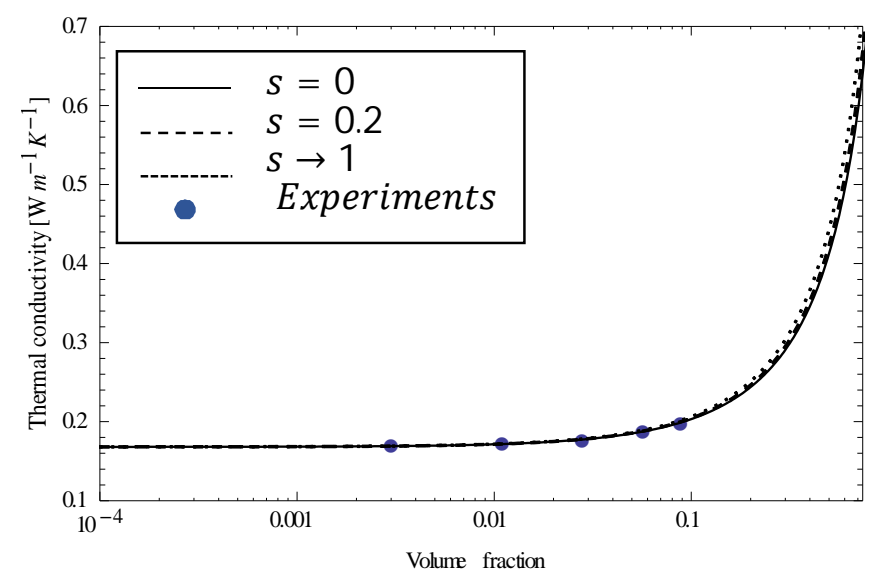

Fig. 6: Effective thermal conductivity of $\mathrm{SiO}_{2}$-epoxyresin as a function of the volume fraction $(\varphi)$ of $\mathrm{SiO}_{2}$ particles for different $s$ values with radius $a_{p}=10 \mathrm{~nm}$. Experimental observations at the same radius (see Ref. 22) are shown in filled circles.

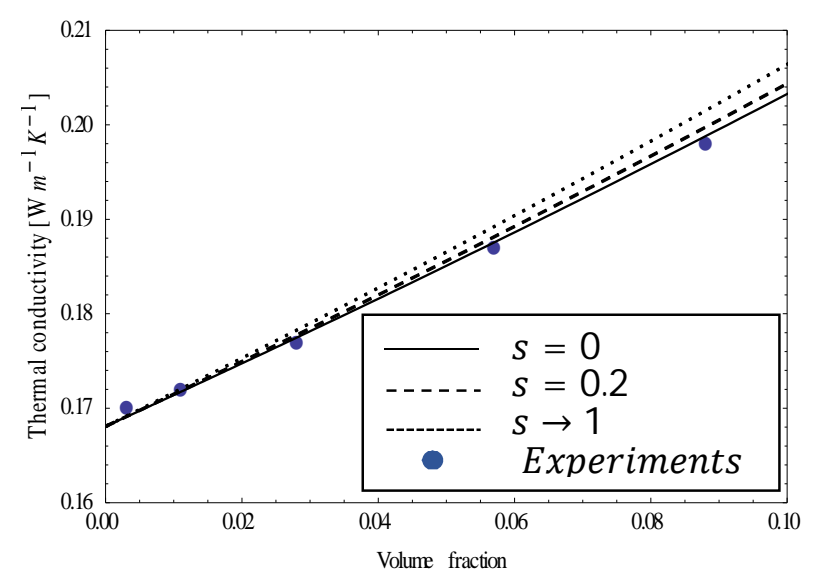

Fig. 7: Effective thermal conductivity of $\mathrm{SiO}_{2}$-epoxyresin as a function of the volume fraction $(\varphi)$ of $\mathrm{SiO}_{2}$ particles for different $s$ values with radius $a_{p}=10 \mathrm{~nm}$, zooming between $\varphi=0$ and 0.1. Experimental observations (see Ref. 22) are shown in filled circles for $a_{p}=10 \mathrm{~nm}$.

Good agreement is obtained between our model and the experimental data. It appears that for this system the influence of specularity is very small but nevertheless $s=0$ gives the best results (Fig. 7). Another interesting effect is that the effective thermal conductivity is now increasing with the volume fraction, while decreasing for $\mathrm{Si}-\mathrm{Ge}$.

\section{Discussion and Conclusion}

To study heat conduction in heterogeneous media composed out of spherical nanoparticles dispersed into a homogeneous matrix, we have followed a route combining the effective-medium approach with extended irreversible thermodynamics. We have studied the influence of several parameters on the effective heat conductivity $\left(k^{\text {eff }}\right)$ and validated our model against others, Monte-Carlo simulations and experimental observations for three different nanocomposites. It is shown that for $\mathrm{Si}-\mathrm{Ge}$, the value of $k^{\text {eff }}$ decreases with the volume fraction of nanoparticles, while the opposite is observed for $\mathrm{SiO}_{2}-$ epoxy-resin. In the $\mathrm{Cu}-\mathrm{Si}$ system, both behaviors are observed depending on the particle size and volume fraction. A larger particle radius $\left(a_{p}\right)$ and a higher surface specularity result into a higher $k^{e f f}$. Larger $a_{p}$ and $s$-values will tend to decrease the particle-matrix interface, which is the main obstacle that the phonons encounter in the nanocomposites. As such, the phonons experience less obstacles and the thermal conductivity increases.

Good accord is obtained between our model and other theoretical ones as well as with experimental data and Monte-Carlo simulations. Our analysis has shown that diffuse scattering is dominant, though some specular scattering can still be present. A reduction of the thermal conductivity as observed in $\mathrm{Si}-\mathrm{Ge}$ is also found in other systems, for instance ErAs nanoparticles in $\mathrm{In}_{0.53} \mathrm{Ga}_{0.47 .}{ }^{3}$ In contrast, in $\mathrm{SiO}_{2}$-epoxy resin composite, $k^{e f f}$ is increasing with the nanoparticle volume fraction. Such a behavior may be interpreted by the much smaller value of the dimensionless $\alpha$ parameter in the case of $\mathrm{SiO}_{2}$-epoxy resin than for $\mathrm{Si}$ $\mathrm{Ge}$, due to a smaller mean free path.

No general rule concerning the behavior of the effective thermal conductivity with respect to the volume fraction can be drawn; indeed, it may either increase or decrease depending on the particle radius, the characteristics of its surface, and the thermal boundary resistance.

For the sake of completeness, we would like to briefly comment about two papers akin to the present one wherein the authors used a hierarchy of fluxes similar to the relations (11) and (12). In the first work, Alvarez and Jou (see Ref. 23) establish an expression of the heat conductivity of a thin layer of thickness smaller than the mean free path of phonons. In the second one, Alvarez 
Effective Thermal Conductivity of Spherical Particulates Nanocomposites: Comparison with Theoretical Models, Monte Carlo Simulations and Experiments

et al (see Ref. 24) generalize their approach to the problem of heat conduction in superlattices formed by the succession of layers of $\mathrm{Si}$ and $\mathrm{Ge}$. There are two main differences with the present work. First, the geometries are different and more particular: plane layers instead of spheres embedded in a matrix. Second, Alvarez and collaborators (see Refs. 23 and 24) assume that all the mean free paths $l_{n}$ are equal, whatever the order $n$ from which follows that they obtain an expression slightly different from (16) given by

$k_{p}=\frac{k_{p}^{0}}{2 \pi^{2} K n^{2}}\left[\sqrt{1+4 \pi^{2} K n^{2}}-1\right]$.

In the future, it is our purpose to generalize the present study to other shapes as cylindrical or ellipsoidal geometries, extending the study also to porous media. Another important effect to be analyzed next is the particles agglomerations which may play an important role. Moreover, in this work, it was assumed that the spheres are randomly distributed, it would be interesting to consider more specific configurations as for instance spheres distributed in a regular array.

\section{List of symbols}

\begin{tabular}{|c|c|}
\hline$a_{p}$ & Particle radius [nm] \\
\hline$a_{p, s}$ & Particle specular radius $[\mathrm{nm}]$ \\
\hline C & Specific heat capacity $\left[\mathrm{MJ} \mathrm{K}^{-1} \mathrm{~m}^{-3}\right]$ \\
\hline$k$ & Thermal conductivity $\left[\mathrm{W} \mathrm{m}^{-1} \mathrm{~K}^{-1}\right]$ \\
\hline $\boldsymbol{k}$ & Wavenumber $\left[\mathrm{m}^{-1}\right]$ \\
\hline Kn & Knudsen number \\
\hline$l$ & Correlation length $[\mathrm{m}]$ \\
\hline$q$ & Heat flux $\left[\mathrm{W} / \mathrm{m}^{2}\right]$ \\
\hline$Q$ & Higher order flux \\
\hline $\boldsymbol{r}$ & Position vector $[\mathrm{m}]$ \\
\hline$R$ & Thermal resistance $\left[\mathrm{m}^{2} \mathrm{~K} / \mathrm{W}\right]$ \\
\hline$t$ & Time $[\mathrm{s}]$ \\
\hline$T$ & Temperature $[\mathrm{K}]$ \\
\hline$v$ & Phonon group velocity $[\mathrm{m} / \mathrm{s}]$ \\
\hline \multicolumn{2}{|c|}{ Greek symbols } \\
\hline$\alpha$ & Dimensionless thermal resistance \\
\hline$\alpha_{i}$ & Phenomenological relaxation time \\
\hline$\beta_{i}$ & Phenomenological correlation length \\
\hline$\Lambda$ & Mean free path $[\mathrm{nm}]$ \\
\hline$\mu_{i}$ & Phenomenological transport coefficient \\
\hline$\varphi$ & Volume fraction $[-]$ \\
\hline$\omega$ & Frequency \\
\hline
\end{tabular}

Superscripts

$0 \quad$ Base state of the bulk material

eff Effective

(n) $\quad \mathrm{N}^{\text {th }}$ order flux

$\wedge \quad$ Fourier transform

Subscripts

$b \quad$ Bulk phonons

coll Collisional phonons at interface

m Matrix

$p \quad$ Particle

ref Reference state

\section{Acknowledgments}

We thank the BelSPo for financial support.

\section{References}

1. D.C. Moreira, L.A. Sphaier, J.M.L. Reis, L.C.S. Nunes, J. Exp. Therm. Fluid Sc. 35, 1458 (2011).

2. J. Chen, S.L. Li, Z.L. Tao, L.Z. Zhang, Int. J. Nanosci. 1, 295 (2002).

3. K.K. Choudhary, D. Prasad, K. Jayakumar, D. Varshney, Int. J. Nanosci. 8, 551 (2009).

4. D. Jou, J. Casas-Vazquez, G. Lebon, Extended Irreversible Thermodynamics, 4th edn. (Springer, Berlin, 2010).

5. G. Lebon, H. Machrafi, M. Grmela, Ch. Dubois, Proc $R$ Soc A 467, 3241 (2011).

6. C.-W. Nan, R. Birringer, D.R. Clarke, H.Gleiter, J. Appl. Phys. 81, 6692 (1997).

7. J.C. Maxwell, Treatise on Electricity and Magnetism, $2^{\text {nd }}$ edn. (Clarendon, Oxford, 1881).

8. D. Bruggeman, Anal. Phys. 24, 636 (1935).

9. A. Minnich, G. Chen, Appl. Phys. Lett. 91, 073105 (2007).

10. G. Chen, Phys. Rev. B, 57, 14958 (1998).

11. A. Behrang, M. Grmela, C. Dubois, S. Turenne, P.G. Lafleur, J. Appl. Phys. 114, 014305 (2014).

12. J. Ordonez-Miranda, R. Yang, J.J. Alvarado-Gil, Appl. Phys. Lett. 98, 233111 (2011).

13. C. Cattaneo, Sulla conduzione del calore Atti del Seminario Matematico e Fisico delle Università di Modena (1948) Vol. 3, p. 83.

14. W. Dreyer, H. Struchtrup, Continuum. Mech. Thermodyn.3, 3 (2003).

15. S. Hess, Z. Naturforsch. 32a, 678 (1977).

16. G. Chen, Int. J. Therm. Sci. 39, 471 (2000).

17. T. Zeng, G. Chen, J. Heat Transfer 123, 340 (2001).

18. H.M. Duong, N. Yamamoto, K. Bui, D.V. Papavassiliou, S. Maruyama, B.L. Wardle, J. Phys. Chem. 114, 8851 (2010).

19. R.H. Perry, D.W. Green, J.O. Maloney, Perry's Chemical Engineers' Handbook, 7th edn. (McGrawHill, New York, 1997) 
20. J. Ordonez-Miranda, J.J. Alvarado-Gil, Comp. Sc. Tech. 72, 853 (2012).

21. M.-S. Jeng, R. Yang, D. Song, G. Chen, J. Heat Transfer 130, 042410 (2008).

22. R. Kochetov, A. V. Korobko, T. Andritsch, P. H. F. Morshuis, S. J. Picken, J. J. Smit, J. Phys. D: Appl. Phys. 44, 395401 (2011).

23. F.X. Alvarez, D. Jou, Appl. Phys. Lett. 90, 083109 (2007).

24. F.X. Alvarez, X. Alvarez-Quintana, D. Jou, J. Rodriguez- Viejo, J. Appl. Phys. 107, 084303 (2010). 